
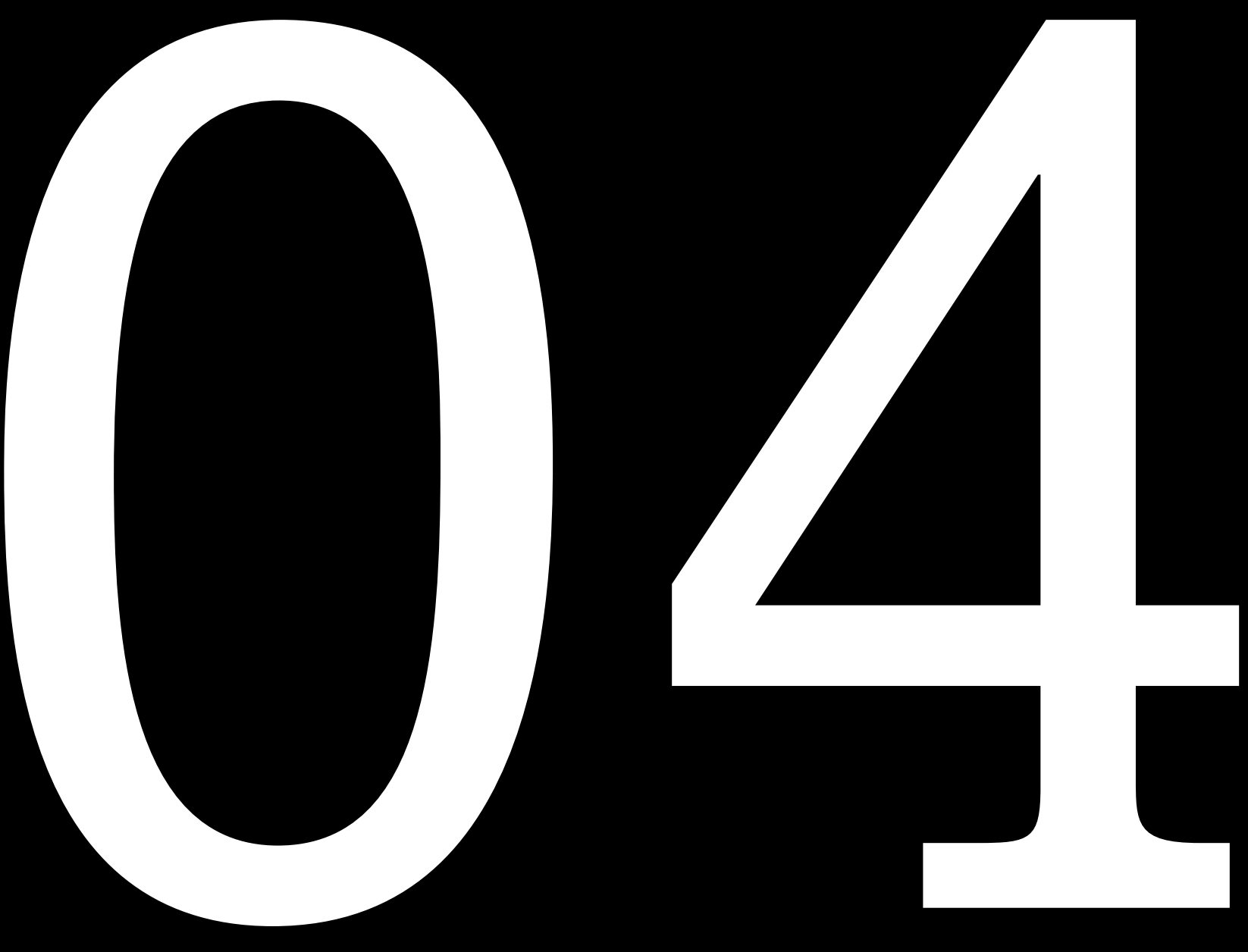

DOI: https://doi.org/10.14483/2422278X.14178 
Cartografiando la

realidad comunitaria:

un acercamiento a las

voces de la comunidad

La Fortaleza ${ }^{1}$

Mapping community reality: An approach to voices of La Fortaleza community

Mapeamento da realidade comunitária: uma abordagem às vozes da comunidade de

\section{La Fortaleza}

\section{Cristhian Adrián García Vergara ${ }^{2}$ Nydia María Rincón Villamizar ${ }^{3}$}

Para citar este artículo: García, C. A. y Rincón, N. M. (2019). Cartografiando la realidad comunitaria: un acercamiento a las voces de la comunidad La Fortaleza. Ciudad Paz-ando, 12(2), 45-59. doi: https://doi.org/10.14483/2422278X.14178

Fecha de recepción: 1 de diciembre de 2018

Fecha de aprobación: 11 de septiembre de 2019

\footnotetext{
1 Artículo de investigación producto del estudio: “La ciudadanía: un abordaje desde las representaciones sociales de los habitantes de la comunidad La Fortaleza, en Cúcuta” apoyado por el Grupo de Investigación en Problemas Socioeconómicos Regionales y Fronterizos Categorizado por Colciencias en C, de la Universidad Francisco de Paula Santander.

2 Trabajador social, especialista en Práctica pedagógica. Miembro del Grupo de investigación en problemas socioeconómicos regionales y fronterizos. Universidad Francisco de Paula Santander, Cúcuta. Correo electrónico: adrigar100@gmail.com, ORCID: https://orcid.org/0000-0002-6747-7327 3 Magíster en Educación, licenciada en Educación con énfasis en Lenguas Modernas. Docente del Departamento de Educación, Artes y Humanidades. Directora del Grupo de investigación en problemas socioeconómicos regionales y fronterizos. Directora del programa de specialización en Práctica Pedagógica. Universidad Francisco de Paula Santander, Cúcuta. Correo electrónico: aprendizaje2020@gmail.com, ORCID: https://orcid. org/0000-0001-7016-8069
} 


\section{RESUMEN}

El presente escrito plantea los hallazgos de la cartografía social (CS) realizada en el asentamiento humano La Fortaleza buscando el reconocimiento del territorio, los recursos activos y las limitaciones sociales con las que conviven. La postura teórica se fundamenta en conceptos como calidad de vida, vulnerabilidad y libertades fundamentales del enfoque de capacidades humanas propuesto por Amartya Sen. La metodología fue de tipo cualitativo con enfoque fenomenológico, lo cual posibilitó el reconocimiento de los actores comunitarios, institucionales como ausentes, las problemáticas sociales y estructurales, y los hechos referenciales que caracterizan a la comunidad La Fortaleza como las implicaciones a nivel social, familiar y comunitario. Es importante considerar que este apartado de resultados, si bien hace parte de un estudio de representaciones sociales frente a un objeto de estudio definido, solo evidencia las implicaciones del contexto social sobre la vida de los habitantes comunitarios.

Palabras clave: calidad de vida, cartografía social, libertades, vulnerabilidad.

\section{ABSTRACT}

This paper presents the findings of the social mapping constructed in the human settlement of La Fortaleza with an aim to recognise the area, its active resources and social limitations encountered by them. The study's theoretical position is formulated on the basis of concepts such as quality of life, vulnerability and fundamental freedoms of the Human Capability approach proposed by Amartya Sen. A qualitative methodology with a phenomenological approach was employed, allowing the authors to recognise community actors, institutional actors, social and structural problems and the referential facts that characterise La Fortaleza community, alongside its social, family and community implications. Notably, the results of the study, while being a part of a social representations' study as opposed to a defined objective of the study, only demonstrates the implications within the community's social context.

Keywords: quality of life, social mapping, freedoms, vulnerability.
Este artigo apresenta os achados da Cartografia Social realizada no assentamento humano La Fortaleza buscando o reconhecimento do território, dos recursos ativos e das limitações sociais com os quais convivem. A posição teórica baseia-se em conceitos como qualidade de vida, vulnerabilidade e liberdades fundamentais da abordagem das Capacidades Humanas proposta por Amartya Sen. A metodologia foi de tipo qualitativo com enfoque fenomenológico, o que possibilitou o reconhecimento dos atores comunitários, institucionais como ausentes, os problemas sociais e estruturais, e os fatos referenciais que caracterizam a comunidade La Fortaleza como as implicações em nível social, familiar e comunitário. É importante considerar que esta seção de resultados, embora faça parte de um estudo de Representações Sociais diante de um objeto de estudo definido, apenas evidencia as implicações do contexto social na vida dos habitantes da comunidade.

Palavras-chave: qualidade de vida, mapeamento social, liberdades, vulnerabilidade. 


\section{Introducción}

Si se considera la perspectiva de desarrollo humano planteada por Sen (1998: 2000), vinculado al disfrute y expansión de las libertades instrumentales como orientación en el análisis de la realidad social de los territorios, permite entrever una radiografía social integral sobre las condiciones de vida de las personas. Condiciones de vida que se ven enfrentadas a un debilitamiento de los vínculos de inserción comunitaria (Aguiló, 2006), de los procesos de individualización (Bauman, 2011), además de un agravamiento-aumento de las situaciones de pobreza y exclusión social (De Robertis, 2000), y de los problemas relativos a las condiciones de vida, es decir, ligados a las desigualdades de género, a la inmigración y al envejecimiento (Giddens, 2007).

En línea de lo anterior, La Fortaleza es un asentamiento humano, ubicado en el Anillo Vial occidental que se encuentra inmerso en un ecosistema terrestre, desértico, carente de arborización (Figura 1) y organizado por sectores (San Miguel, Alto de los Padres, Los Pinos, El Mirador, El Plan, Las Iglesias, La Escuela, El Paraíso y Buena Vista). A nivel general, la comunidad está conformada por familias desplazadas por la violencia, las situaciones socioeconómicas de la región y la crisis migratoria de la frontera colombo-venezolana. Al respecto, estudios ratifican las condiciones de vulnerabilidad y de calidad de vida de los habitantes comunitarios frente a las falencias de habitalidad, el acceso a la salud, la educación, los servicios públicos y la vivienda, "comprendiendo con ello no sólo la existencia o no de estos aspectos en la vida de los habitantes sino la calidad de las condiciones de los mismos y las formas mediante las cuales pueden tener accesibilidad a estos" (Leal et al., 2018, p. 52).
Considerando lo expuesto, se realizó la Cartografía Social para conocer las necesidades sociales y sus implicaciones en la vida de los habitantes comunitarios de La Fortaleza, reconociendo un contexto social desde sus limitaciones como recursos activos de la comunidad. De esta forma, se realiza una apuesta de análisis de las condiciones de vida desde el enfoque de las capacidades humanas de Amartya Sen (2000) ligando cada una las formas de libertades fundamentales y generando un concepto social desde el trabajo social hacia la comunidad.

\section{Base teórica}

Estudiar el contexto social de una comunidad desde las voces de sus habitantes involucra nociones conceptuales como calidad de vida, vulnerabilidad y libertades fundamentales. En este sentido, entender el concepto de calidad de vida ${ }^{4}$ parte por reconocer su multidimensionalidad, en la cual se integran componentes sustantivos como la salud, la economía, el trabajo, la vivienda, las relaciones sociales, el ocio, el medio ambiente, los derechos, así como también: "el juicio subjetivo individual y colectivo, acerca del grado en el que se ha alcanzado la satisfacción de necesidades" (Barranco, 2009 p. 134).

Ahora bien, el concepto de vulnerabilidad, en palabras de Lampis (2011), es entendida como una función inversa que restringe o limita a individuos, grupos o comunidades en su satisfacción de necesidades y goce de bienestar social, lo que implica una pérdida de activos materiales e inmateriales. Asímismo, es valioso considerar que las personas, grupos o comunidades tienen capacidades para $4 \quad$ Para mayor profundización teórica e histórica, consultar el trabajo
realizado por Tovar (2010).

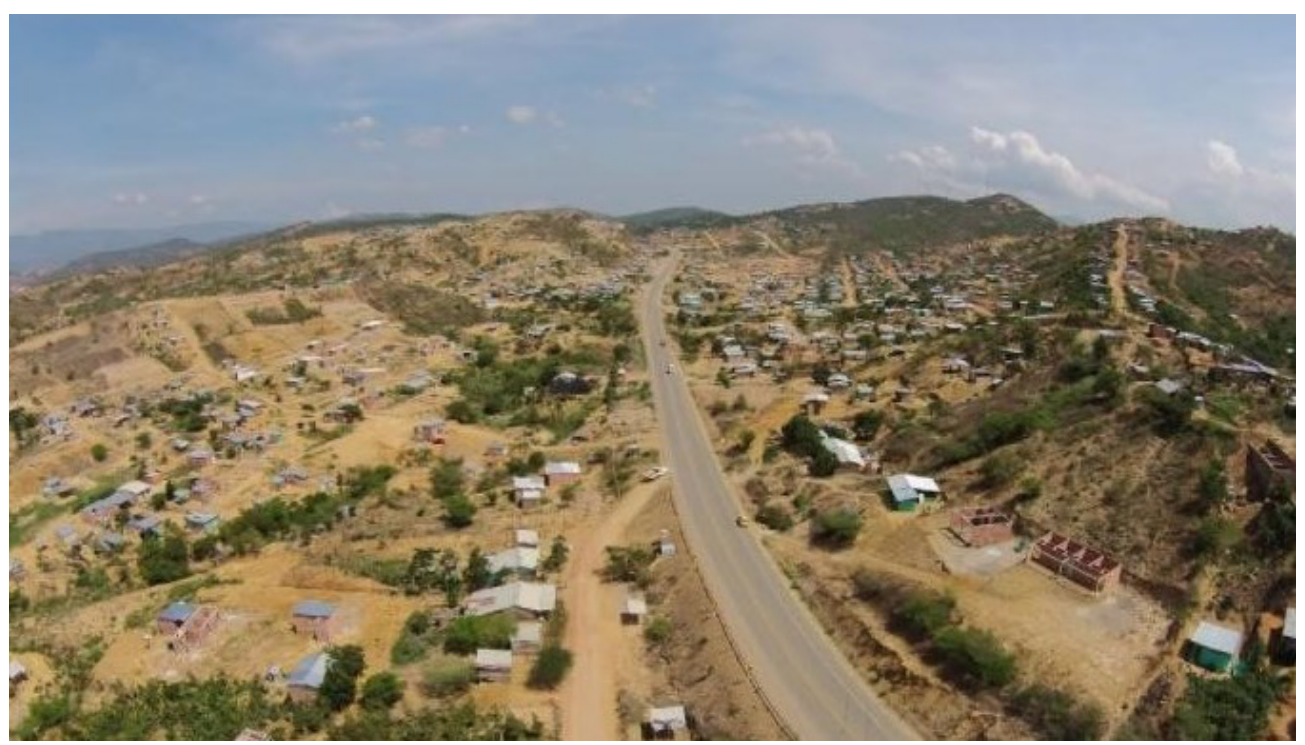

Figura 1. Comunidad La Fortaleza Fuente: Google Satelital. 
hacer frente desde su conjunto de posibilidades y activos a situaciones críticas (Ospina y Egea, 2018).

En este contexto, Amartya Sen (2000) propone un nuevo enfoque del desarrollo, entendido no solo desde el aspecto económico, sino también cultural, social y político, referido "como un proceso de expansión de las libertades fundamentales que disfrutan los individuos" (p. 19). conceptualizando a las libertades como aquellas capacidades que poseen los individuos, grupos o comunidades de conseguir condiciones de vida que consideran valiosos para sí y su entorno. Por lo tanto, las consideraciones teóricas y normativas del enfoque de las Capacidades Humanas permiten analizar la calidad de vida en función de las libertades efectivas que gozan los individuos (Ramírez, Arias y Marulanda, 2016).

En función de lo anterior, Sen (2000) plantea cinco libertades fundamentales como, estas son: la libertad política, ligada a los derechos políticos y a su capacidad de ejercicio por el ser humano; la libertad económica, referenciada a la oportunidad de consumir, intercambiar y producir como participar en el mercado; las libertades sociales, consideradas como los elementos necesarios para la calidad de vida y el bienestar en general; la libertad como garantía de transparencia, entendida como el grado mínimo de confianza en la red social, cultural y economía en un escenario específico, y, finalmente, la libertad como seguridad protectora.

Las libertades fundamentales descritas no funcionan como islas separadas, sino como una red interconectada donde la afectación de una restringe o priva la libertad de las demás. Es claro precisar, que el desarrollo de las libertades involucra una sinergia con el conjunto de elementos que tiene el contexto para potenciarlas o para restringirlas. En este proceso, las contradicciones sociales pueden ser tomadas como estructurales (pobreza, desigualdad, corrupción, entre otros) y no estructurales (vistos en la dinámica cotidiana de los ciudadanos como discriminación, violencia basada en género, consumo de sustancias psicoactivas, entre otros), no obstante, ambas restringen las libertades de una persona en su contexto.

\section{Metodología}

La investigación se suscribe desde lo cualitativo al permitir la comprensión de una realidad social desde las voces de sus involucrados. Al respecto, autores como Sampieri, Collado y Baptista (2014) mencionan que la investigación cualitativa permite "describir, comprender e interpretar los fenómenos, a través de las percepciones y significados producidos por las experiencias de los participantes" (p. 11). En convergencia, se asumió el enfoque fenomenológico (Schütz, 1972), ya que se le brinda importancia al saber cotidiano de cada uno de los habitantes comunitarios participantes, en la escritura de cada uno de los signos y símbolos como en sus interpretaciones sociales.

Por otra parte, como herramienta metodológica se empleó la cartografía social (CS) para reconocer el contexto social de los habitantes de La Fortaleza a partir de las voces de cada uno de los participantes. En tal sentido, la CS se entiende como: "una metodología participativa y colaborativa de investigación que invita a la reflexión, organización y acción alrededor de un espacio físico y social especifico" (Vélez, Rátiva y Varela, 2012, p. 62) que permite descubrir y tocar lo invisible y valorar y reordenar lo visible (Restrepo, Velasco, y Preciado, 1999) desde el diálogo sobre la comunidad y sus dinámicas cotidianas. A continuación, se sintetiza el acercamiento metodológico de la CS aplicado a la comunidad La Fortaleza (Tabla 1).

\section{Tabla 1. Constructo metodológico de CS}

Taller - Cartografía Social

\begin{tabular}{ll}
\hline Definición de objetivo & $\begin{array}{l}\text { Reconocer el contexto social donde viven y conviven los habitantes comunitarios de La Fortaleza } \\
\text { frente a sus problemas sociales y recursos activos. }\end{array}$ \\
\hline Población participante & Los habitantes comunitarios de La Fortaleza. \\
\hline $\begin{array}{l}\text { Definición de escala } \\
\text { temporal y espacial }\end{array}$ & La comunidad La Fortaleza: desde su pasado, presente y futuro. \\
\hline Definición de temática & Problemas sociales y recursos comunitarios. \\
\hline $\begin{array}{l}\text { Elección de la persona } \\
\text { relatora }\end{array}$ & $\begin{array}{l}\text { Dos profesionales de apoyo en cada subgrupo de la CS. Se graba cada discusión acaecida en el } \\
\text { grupo para su posterior análisis categorial. }\end{array}$ \\
\hline $\begin{array}{l}\text { Convenciones o } \\
\text { simbología }\end{array}$ & $\begin{array}{l}\text { Se han construido de acuerdo a los símbolos propuestos por el grupo a través de preguntas } \\
\text { orientadoras frente a los actores comunitarios e institucionales, los problemas sociales y } \\
\text { estructurales y los hechos referenciales. seguidamente se ubicaron en los mas y se } \\
\text { diálogo de saberes en aras de conocer las implicaciones de cada una de ellas en las condiciones } \\
\text { de vida de los habitantes comunitarios (Figura 2). }\end{array}$ \\
\hline Socialización & $\begin{array}{l}\text { Finalizado el mapa social y las relaciones de cada convención, cada grupo socializa su mapa y se } \\
\text { retroalimenta con los saberes del otro grupo. }\end{array}$
\end{tabular}

Fuente: elaboración propia ajustada a las consideraciones de Barragán-León (2018). 


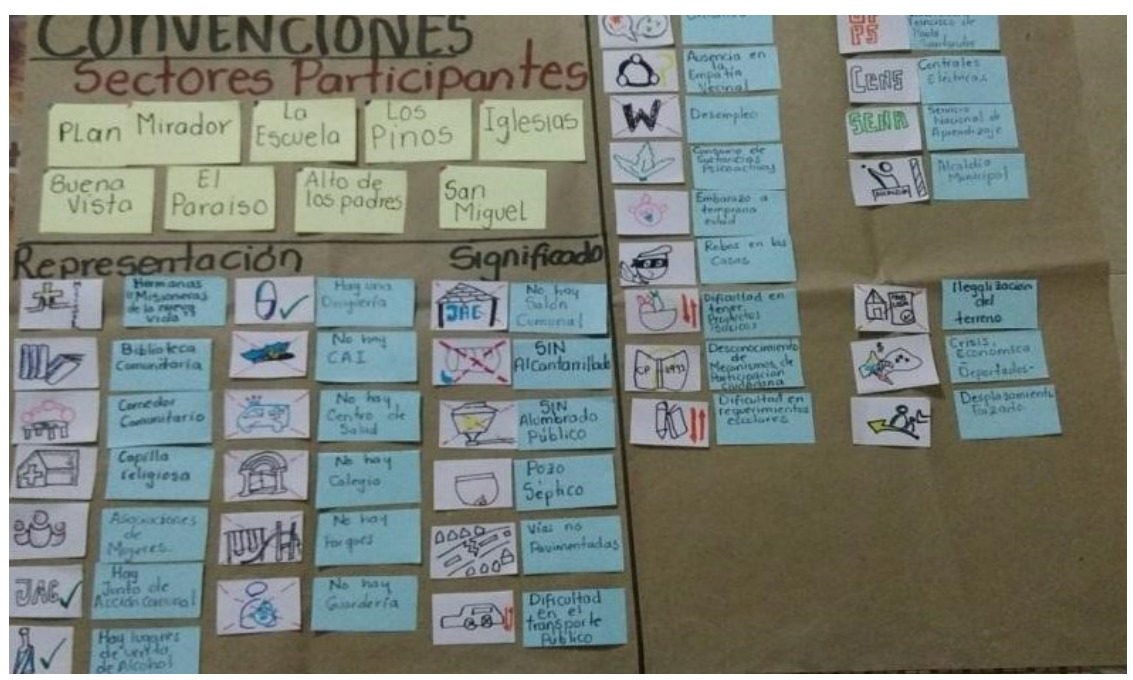

Figura 2. El mapa refleja las problemáticas de la comunidad a través de las convenciones Fuente: elaboración propia.

Por su parte, se analizó la información con el apoyo de la metodología de "codificación abierta" de Strauss y Corbin (1998). El proceso de decodificación lo integran (siguiendo el planteamiento de Cuñat, 2007): la decodificación abierta, lo primero en efectuarse fue la trascripción de los diálogos de cada grupo de la CS; adicionalmente, se realizó una codificación simple a cada sujeto comunitario participantes. Los códigos están coordinados con la CS y un número consuetudinario 01 (representa a un habitante comunitario que participó en la construcción del contexto comunitario).

Luego, se analizó línea por línea cada idea que aportaron los participantes, ayudando a identificar categorías iniciales, preliminar de todos los conceptos. Se prosiguió con la decodificación axial, en donde se seleccionaron los conceptos más relevantes y similares para cada categoría de la codificación abierta. Consecutivamente, se agruparon categorías centrales, que a la vez estaban compuestas por subcategorías. Seguidamente, se extrajeron de las entrevistas los datos que los participantes aportaron, sin realizar modificación alguna, integrándose en cada una de las categorías finalmente seleccionadas.

Por último, se realizó la decodificación selectiva, en donde se seleccionaron los conceptos más relevantes resaltados en las diferentes categorías que ayudaron a comprender los hallazgos frente el contexto social de la comunidad. Tomando en cuenta lo planteado en la metodología y el análisis de los resultados, la Tabla 2 muestra los resultados categoriales producto del análisis.

\section{Hallazgos}

A través de la CS, los habitantes comunitarios hicieron el trazado de su territorio reconociendo los actores comunitarios, institucionales y ausentes; las problemáticas sociales y estructurales, y los hechos referenciales. Características comunes de la comunidad La Fortaleza, permitiendo una radiografía social integral de la dinámica en el asentamiento humano.

\section{Actores comunitarios}

En el marco de la categoría analítica "actores comunitarios", emergen la biblioteca comunitaria dirigida por las Hermanas Misioneras de la Nueva Vida, que se ha venido consolidando en un espacio de protección para los Niños, Niñas, Adolescentes y Jóvenes (NNAJ) de la comunidad hacia fenómenos que limitan su proyecto de vida como el reclutamiento a bandas criminales o hacia los problemas sociales identificados en la primera parte de la CS como el consumo de sustancias psicoactivas o el embarazo adolescente. En opinión de los involucrados:

Si de pronto también por la misma falta de educación o falta de qué hacer, por ejemplo, nosotras también buscamos el espacio de que los niños estén ocupados ya sea en la biblioteca o en música o en deporte para que no caigan en las drogas o en las bandas criminales. (CS01)

La biblioteca comunitaria nos ayuda bastante, mis hijos van allá por las tareas y porque también les enseñan las hermanas, hacer bien las cosas y no andar en malos pasos [...] uno de mis hijos está en el futbol y eso, así está ocupado, es que a veces se quedan solos en casa, yo me tengo que ir a buscar trabajo y a veces me sale una que otra cosita por hacer. (CSO9)

Con lo anterior, se rescatan actividades de lectura, deporte y música como alternativas de ocupación del tiempo libre de los niños, niñas, adolescentes y jóvenes que se convierten en estrategias comunitarias para mitigar o prevenir los distintos riesgos sociales a los que se enfrenta cotidianamente la población en la comunidad.

En otra instancia, algunos participantes mencionaban cómo la capilla religiosa se convierte en un espacio para 
Tabla 2. Contexto social de la comunidad La Fortaleza: CS

\section{CARTOGRAFÍA SOCIAL}

\begin{tabular}{|c|c|c|}
\hline Categoría & Categoría emergida & Reflexión comunitaria \\
\hline & $\begin{array}{l}\text { La biblioteca como espacio de } \\
\text { protección de los NNAJ }\end{array}$ & Ocupaciones a través de la lectura, la música y el deporte \\
\hline & $\begin{array}{l}\text { La Capilla religiosa como espacio } \\
\text { de fortalecimiento espiritual y } \\
\text { comunitario }\end{array}$ & $\begin{array}{l}\text { Invitación a la sana convivencia y a la resolución asertiva de los conflictos. } \\
\text { Actos de fe y oración para buscar buen conocimiento en la relación con } \\
\text { el otro y la otra. }\end{array}$ \\
\hline & $\begin{array}{l}\text { La asociación como empoderamiento } \\
\text { de la mujer comunitaria }\end{array}$ & $\begin{array}{l}\text { Participación activa en los asuntos comunitarios en búsqueda de } \\
\text { soluciones }\end{array}$ \\
\hline \multirow[t]{4}{*}{ Actores comunitarios } & $\begin{array}{l}\text { La Junta de Acción Comunal } \\
\text { como Referencia de Organización } \\
\text { Comunitaria }\end{array}$ & $\begin{array}{l}\text { Resolución de problemas comunitarios: Adquisición de los lotes } \\
\text { Procesos de legalización. }\end{array}$ \\
\hline & & La obtención de los servicios públicos \\
\hline & $\begin{array}{l}\text { Las implicaciones de la legalización de } \\
\text { la comunidad }\end{array}$ & Consumo consiente de la Energía eléctrica en los hogares \\
\hline & & $\begin{array}{l}\text { Falta de alumbrado público aumenta la delincuencia y el consumo de } \\
\text { sustancias psicoactivas }\end{array}$ \\
\hline Actores institucionales & $\begin{array}{l}\text { Las implicaciones en el fortalecimiento } \\
\text { de la convivencia social }\end{array}$ & $\begin{array}{l}\text { Proyecciones sociales y capacitaciones en resolución asertiva de los } \\
\text { conflictos }\end{array}$ \\
\hline Actores Ausentes & $\begin{array}{l}\text { Las implicaciones de la ausencia } \\
\text { institucional }\end{array}$ & Aumento de la inseguridad por falta de presencia de agentes de policía \\
\hline \multirow[b]{5}{*}{$\begin{array}{l}\text { Problemáticas sociales } \\
\text { y estructurales }\end{array}$} & La informalidad y desempleo como & \\
\hline & deterioro de la dinámica familiar & Aumento del conflicto intrafamiliar \\
\hline & $\begin{array}{l}\text { La inseguridad del contexto como } \\
\text { peligro de la dignidad de los habitantes }\end{array}$ & $\begin{array}{l}\text { Robos, intolerancias en el asunto de definir los límites de los lotes, } \\
\text { aumento de la drogadicción }\end{array}$ \\
\hline & La pobreza como límite de la libertad & Dificultad en el acceso a requerimientos escolares y alimenticio \\
\hline & $\begin{array}{l}\text { Ausencia de la empatía vecinal } \\
\text { como baja resolución asertiva de los } \\
\text { conflictos }\end{array}$ & $\begin{array}{l}\text { Violencia primaria entre los vecinos } \\
\text { Individualidad, apatía hacia los sucesos del otro y la otra }\end{array}$ \\
\hline \multirow[b]{4}{*}{ Hechos referenciales } & $\begin{array}{l}\text { La ilegalidad del Asentamiento } \\
\text { Humano como falta de oportunidades }\end{array}$ & Historia de la comunidad \\
\hline & $\begin{array}{l}\text { El desplazamiento forzado y crisis } \\
\text { económica como consecuencia de la } \\
\text { violencia }\end{array}$ & Inicio de una nueva historia \\
\hline & $\begin{array}{l}\text { La justicia encabezada por grupos } \\
\text { armados irregulares }\end{array}$ & $\begin{array}{l}\text { Grupos armados ilegales ejercen control en la comunidad (son } \\
\text { invisibilizado por temores infundados). }\end{array}$ \\
\hline & $\begin{array}{l}\text { La comunidad vulnerable como } \\
\text { escenario de proselitismo político }\end{array}$ & Uso y abuso de la comunidad para las campañas electorales \\
\hline
\end{tabular}

Fuente: elaboración propia.

fortalecer la resolución de conflictos a través de la mediación de las Misioneras de la Nueva Vida y el Sacerdote:

Él dice (el sacerdote) que él se siente muy mal y que es muy duro que siga pasando cosas así raras, que debemos orar y pedirle a Dios que nos ayude y que ponga un buen conocimiento a esa que tiene la mala intención, porque no tiene temor [...] yo tengo mucho temor a Dios, siempre que tengo algún problema voy a la capilla [...] a mí me enseñaron a trabajar, a respetar muchos valores como en vez de tener que quitarle a otro mejor hay que darle. (CS06)

Por otra parte, los participantes mencionan cómo la organización de las mujeres, a través de asociaciones, busca empoderarlas para mejorar su calidad de vida y la de su entorno. Uno de los participantes mencionó que: 
Contamos con 2 asociaciones de mujeres reconocidas una se llama ASOMUCOMFOR, la asociación de mujeres con fortaleza y las CHICAS F [...] cada asociación tiene un grupo de 30 mujeres que trabajan, tienen talleres y van funcionando muchas cosas, entonces esta comunidad va creciendo y sobre todo va viendo uno el deseo de superación de muchas mujeres. (CS05)

De esta forma, una de las habitantes participantes reconoció su liderazgo en la asociación planteando lo siguiente; "A mí me registraron en una asociación, para solicitar ayudas en el barrio como acá no tenemos ni luz ni agua propia entonces pues todo es ilegal. Por eso estoy en la asociación para buscar alguna solución y salir a adelante" (CS07).

La realidad planteada por la participante evidencia el empoderamiento de la mujer a través del liderazgo de las asociaciones de mujeres que ha permitido fortalecer la organización comunitaria, en miras de legalizar el asentamiento humano y conseguir los servicios públicos para los habitantes de los diferentes sectores que hacen presencia.

Los participantes mencionaron cómo la Junta de Acción Comunitaria (JAC) ha posibilitado la organización de la comunidad en términos de conseguir los servicios públicos e ir adelantando la legalización de la comunidad a través de diálogos con la administración municipal y con distintos líderes políticos. Al respecto, consideraron que:

A través de la junta de acción comunal, se ha traído el agua no en un primer momento a veces se dificulta para las partes altas porque la fuerza del agua no es la suficiente y no alcanza a llegar el agua a la parte alta que somos como los más afectados [...] la luz, pues ahora centrales electicas ha querido invertir acá y ya prácticamente postearon todo lo que es La Fortaleza y ya hay un sector de La Fortaleza que se llama el sector el plan donde estamos ahora ubicados, ya estamos contando ahora con la luz propia de centrales, ahí está el contador. (CS05)

En tal sentido, la JAC se ha mostrado, desde la perspectiva comunitaria, como un espacio para resolver, específicamente, los problemas que surgen al momento de definir qué es de quién y cómo se puede resolver, por lo menos, en los temas relacionados con los espacios de la comunidad. Todo ello, ha llevado a considerar a la JAC como un elemento clave para configurarse como comunidad y fortalecerse entre los habitantes comunitarios:

Aunque todavía la JAC no es legal, nos hemos venido organizando para ir teniendo la legalización del barrio. Nosotros no nos metemos con nadie, no queremos problemas con nadie, solamente nosotros asesoramos a la gente que llega a mirar lotes, les mostramos, les explica- mos cómo es la cosa aquí que esto se va hacer legalizar, que el que comprar pues se le respeta que cerque y eso queremos es que llegue gente para tener más comunidad, más apoyo y todo eso. (CS06)

Con la última narrativa se aprecia la ilusión de los habitantes en conseguir la legalización del asentamiento, pues les permite llegar a ser una comunidad con las oportunidades como cualquier otro barrio de la ciudad de Cúcuta, además se visionan como una comunidad unida que se apoyan entre sí.

\section{Actores institucionales y ausentes}

La segunda categoría fueron los actores institucionales reconociendo las implicaciones que ejercen en la legalización de la comunidad (como, por ejemplo, la obtención de energía, formas de ahorro de la electricidad, entre otras formas) y en el fortalecimiento en la convivencia social, como lo realizado por entidades formativas en materia de resolución de conflictos.

En este sentido, se ha identificado el accionar de un actor institucional privado como Centrales Eléctrica realizando procesos de sensibilización en la obtención de la energía eléctrica de forma legal (compra de tarjeta recargable de energía prepagada) y de la importancia de ahorrar: "Si es la luz prepagada entonces la persona recarga y va mirando constantemente cuánto va el consumo, entonces es una manera también para que la gente aprenda a ahorrar y a controlar, no" (CS05).

Por otra parte, los habitantes comunitarios mencionan que la falta de alumbrado público ${ }^{5}$ en la comunidad genera espacios oscuros aprovechados para la delincuencia y el consumo de sustancias psicoactivas, poniendo en riesgo a la comunidad en general, y a los niños, niñas, adolescentes y jóvenes, en particular:

Uno ya no puede pasar por las canchas porque como no hay luz se reúne gente con malos pensamientos, a veces atracan a las personas que pasan tarde de la noche y siempre encuentro jóvenes fumando, aprovechan que está solo y oscuro para cometer fechorías. (CSO7)

Otro actor institucional importante que hace presencia en la comunidad es la universidad ${ }^{6}$ y el Servicio $\mathrm{Na}-$

5 Centrales Eléctrica ha venido avanzando en la instalación del alumbrado en varios sectores de la comunidad, sin embargo, hay sitios específicos donde no hay alumbrado lo cual ha generado inseguridad para los habitantes comunitarios de La Fortaleza.

6 En la comunidad han venido varias Universidades con proyectos de proyección social como la Universidad Francisco de Paula Santander (Pública), Universidad Simón Bolívar (Privada), Universidad Minuto de Dios (Privada) y la Universidad Libre (Privada). Los proyectos implementados han trabajo temáticas como resolución de conflictos, asesorías legales, pedagogía infantil, brigadas de salud, entre otros. 
cional de Aprendizaje ${ }^{7}$ a través de procesos de asesoría y realización de diagnósticos para la identificación de los problemas que aquejan a la comunidad, con el objetivo de trazar la escritura de acciones que permitan mejorar sus condiciones. Adicionalmente, la percepción hacia este actor es positiva por su interés social en el fortalecimiento y desarrollo comunitario, brindando una aceptación por parte de los habitantes. Al respecto mencionan que "Para mí si chévere, porque uno se siente acompañado, que están con uno, que lo quieren ayudar, a mí me gustó mucho" (CS06D). Lo anterior refleja la necesidad latente de la comunidad frente a un acompañamiento integral en la construcción de calidad de vida en los diversos escenarios a nivel comunitario, familiar e individual.

Por otro lado, se evidencian los actores que no hacen presencia en la comunidad como el Comando de Acción Inmediata (CAI), que siendo un actor institucional que brinda garantías de seguridad para la comunidad, ha implicado que:

Hay mucha inseguridad aquí, ellos (la policía) garantizan que haya seguridad, un puesto de policía hace que nos sintamos más seguros frente a los problemas que a diario vivir, no solo con los ladrones, que constantemente saquean los ranchos o casos del vecindario, sino también de los problemas pequeños que se viven en la familia. (CS07)

Adicional al puesto de policía, la falta de un centro de salud que permita la atención de urgencia a los problemas que enfrenta la comunidad en materia de salud y vida, dificulta en muchas ocasiones, que los habitantes puedan acceder. En palabras de uno de los habitantes:

Hay ocasiones en que nos enfermamos, no tenemos a donde ir, y nos toca ir al más cercano que sería en Antonia Santos, a veces es muy lejos, no hay calles pavimentadas, si llueve pues es más difícil salir de aquí [...] tener un puesto de salud es primordial para la comunidad, para que nuestros hijos puedan estar más tranquilos y sanos. (CSO2)

\section{Problemáticas sociales y estructurales}

Dentro de los problemas estructurales que más aquejan a la comunidad se encuentra la informalidad y el desempleo. En relación con esto, se menciona:

Los tipos de familia yo creo que las más numerosas que usted pueda imaginarse. Que muchas viven del rebusque, de vender productos en otros barrios en una ca-

7 El SENA ha llevado ofertas educativas y cursos cortos a la comunidad en aras de fortalecer las habilidades productivas de los habitantes comunitarios. rretilla, en una canasta, en bolsos, muchos pues trabajan en lo que les salgan, entonces es frecuente ver muchas personas por ahí como desempleadas, tanto hombres como mujeres, familias muy numerosas yo creo que la familia que menos hijos tiene son 3 de ahí para adelante 4, 5 y hasta 6. (CS05B).

En razón de lo expuesto, se considera que la mayor parte de la población que vive en La Fortaleza es desempleada o tiene un empleo informal de "rebusque" ${ }^{8}$ como lo denominan los habitantes. Lo anterior posee una serie de implicaciones en la dinámica familiar, por una parte al dejar a los hijos solos en los hogares mientras los padres buscan el diario para sobrevivir, y por otra parte acarrea el aumento de conflictos al interior de los hogares cuando escasean los recursos económicos.

Hay días que uno debe decidir si ir a trabajar o quedarse en casa a cuidar a mis hijos, porque tenemos que comer de algún lado y pues a veces toca salir a buscar el pan [...] las hermanas nos han ayudado bastante con el comedor que tienen, allí van mis hijos [...] si, cuando no hay plata o comida o la cosa esta bien difícil uno se desespera no saber a quién acudir o que hacer, y esa es la situación de muchas familias aquí y eso hace que las peleas en la familia se den, no. (CS09)

Desde la perspectiva de una de las participantes, el desempleo se origina en: "falta de preparación y estudio de las personas para que puedan salir a defenderse, sobre todo las mujeres que somos las más afectadas" (CS07). Con ello, la participante reflexiona en siguiente cita frente a cómo el trabajo dignifica la familia y permite mejorar sus condiciones sociales y económicas para enfrentar los desafíos del diario vivir:

Les cambia todo (tener trabajo), lógicamente, claro porque ya va a haber dinero para hacer mercado, para los hijos, para cubrir las necesidades, además se ve mucho desempleo y la mayoría trabajan de oficios varios, vendiendo pipas, pues puros trabajos que le dan para lo mínimo [...] y es que, con el trabajo, se le puede ofrecer un mejor futuro a los hijos y a la familia en general, por eso es tan importante que el Estado tenga más presente a nosotras. (CS07)

Por otra parte, se identificó la inseguridad que vive la comunidad producto delos constantes robos alas propiedades,

8 Actualmente, no existe una caracterización socioeconómica de la comunidad que refleje datos estadísticos de su ocupación laboral, educativa, satisfacción de necesidades, entre otras. Sin embargo, existe un panorama general de la situación socioeconómica de Cúcuta frente a esas variables que se agudizan en asentamientos humanos con altos índices de vulnerabilidad como La Fortaleza. 
como también de los espacios donde se consume sustancias psicoactivas y aprovechan para realizar actos vandálicos contra los habitantes. Adicionalmente, manifestaron los conflictos que surgen con los lotes y sus limitaciones. Al respecto, se cuestionaron mencionando que:

Y como en todos los barrios también hay inseguridad, hay problemas de drogadicción, hay robos últimamente se han intensificado, casos de intolerancia por las dificultades por los lotes, gente que se pelea por un pedazo de tierra, por una piedra que este tiene y el otro no. (CS05).

Como problema estructural identificado que afecta a gran parte de la comunidad, específicamente a las mujeres cabeza de hogar, son los altos índices de pobreza al no contar con las necesidades básicas satisfechas. Lo anterior, se refleja en las condiciones de salubridad debido a que no hay un puesto de salud en la comunidad y deben trasladarse al barrio más cercano, lo cual les afecta cuando se trata de una situación de emergencia, a lo que se suma la falta de recursos económicos que impide el acceso a una canasta básica de alimentos, el desempleo, las enfermedades que giran en los distintos espacios de la comunidad, y un riesgo social de las adolescentes y jóvenes al quedar embarazadas a temprana edad:

Entonces como son mal alimentados (los niños, niñas y jóvenes en general), no cuentan de pronto con un agua potable, un almacenamiento de esa agua en las mejores condiciones pues muchos problemas de salud, muchísima población embarazada, no más aquí en este barrio en este tiempo con las hermanitas se contabilizaba alrededor de unas 20 mujeres embarazadas. (CS05)

En consecuencia, una de las lideresas comunales, miembro de la asociación de las Chicas F, considera el impacto que genera el consumo de sustancias psicoactivas y el embarazo adolescente, particularmente en los jóvenes de la comunidad, entendiendo que su proyecto de vida se trunca y no permite un avance en su calidad de vida y, además, plantea que es a través de la educación que se pueden generar procesos de cambio:

Los afectan porque se truncan su vida, no estudian no se preparan, pues claro ahí es donde viene más empobrecimiento, porque si uno no estudia pues traes niños a este mundo a sufrir, a pasar necesidades, se incrementa la delincuencia, bueno pues hay de todo (CSO7A)

En el marco de la convivencia social, los participantes plantearon la presencia de acciones de irrespeto e intolerancia hacia las dificultades que se presentan en el trascurrir de la cotidianidad, siendo un catalizador de la resolución no asertiva de los conflictos en la comunidad. Asimismo, se constata el uso de la violencia física por cuestiones familiares o vecinales, como por ejemplo, la definición de los linderos de los lotes, tal y como lo considera una narrativa comunitaria:

Porque el lote no era de ellos, se agarraron a golpes, mejor dicho, casi se matan, es que es verdad, un ejemplo usted quiere venirse para acá y a usted le dijeron ese lote es de tal fulano si, y se metan y construyen y después usted quiera venir a agarrarse con él. (CSO2)

\section{Hechos referenciales}

Como se ha escrito previamente, la comunidad La Fortaleza es un asentamiento humano en cuanto se refiere a la falta de legalización como barrio, asumiendo también el negocio que se ha venido realizando con la venta de los lotes indebidamente apropiados con fines de lucro individual o buscando encontrar una vivienda digna para vivir con la familia:

Pues al principio si fue como invadido por las personas que ya más o menos como hace 3 años tenían riesgo de desalojo, pero ya actualmente no y al principio hay algunos lugares que, pues algunos lotes digámoslo así que se apropiaban, o sea, primero un dueño se apropiaba de varios y después lo iba vendiendo a otro, pues por necesidad. (CSO4)

Continuando con el marco de los hechos referenciales, el conflicto armado ha hecho que millones de colombianos abandonen sus territorios y se desplacen a nuevas regiones, implicando afectaciones al proyecto de vida y un cambio a sus condiciones sociales, económicas y culturales. Los habitantes mencionaron que muchos de sus vecinos son víctimas del conflicto armado $^{9}$, y por tal razón, han tenido que buscar un nuevo lugar donde empezar. También, referencian a personas que, por las condiciones económicas de la ciudad y la crisis fronteriza en general con Venezuela, los ha llevado a buscar un nuevo lugar en búsqueda de vivienda, al respecto mencionan que:

Hay personas que se cansaron de pagar un arriendo y ahora han venido a vivir acá, son personas que también huyen del conflicto, ya sea en primer grado o de manera secundaría como se dice, pero son víctimas de la violencia, huyen al perderlo todo al ver de pronto la violencia muy cerca entonces han querido venirse acá. Acá hay historias bastantes lamentables, las mujeres han perdido a sus esposos o han desaparecido a sus hijos en esos falsos positivos incluso como la situación de Venezuela muchos desaparecidos entonces todo eso pues lo van encontrando unos acá. (CS05)

$9 \quad$ Al respecto, Jesús Ernesto Urbina Cárdenas (2017) plantea los efectos de la guerra en Colombia y su repercusión en la sociedad en general. 
Con lo anterior, en las comunidades vulnerables como es el caso de La Fortaleza, empiezan hacer nido la presencia de actores en búsqueda de conseguir la autoridad e imponer sus mandatos, así lo cuestiona uno de los participantes:

También hay presencia como de un grupo armado, cierto, porque uno no los ve prácticamente, pero eso es mejor como guardar silencio [...] si a veces como que ellos son los que tratan de impartir justicia, entonces, tal vez sea a través de amenazas, cierto, para extorsionar o para sacar o de pronto calmar los ánimos de alguien que le están causando alguna incomodidad aquí en la comunidad. (CS05)

Aunado a lo expuesto, los habitantes comunitarios son vistos como potencial de votos para los candidatos en las elecciones que se realizan, en torno a ello los participantes cuestionaron el uso y abuso que hacen de ellos como comunidad para participar en las votaciones de ciertos candidatos políticos para poder acceder a beneficios 0 promesas, con las que llegan a sus espacios comunitarios:

Pues ya ahorita digamos vamos a empezar como esa etapa de elecciones, de pronto se van venir a presentar diferentes, o sea, como quien dice a ofrecer no viendo de aquí está un lugar de tantas necesidades, entonces vienen a prometer unos y a prometer otros, entonces eso de pronto divide también. (CS04C)

A lo largo del trayecto evocado en las anteriores líneas, se ha reconocido las condiciones sociales y económicas de la comunidad que marcan un punto de partida hacia las posibilidades reales que tiene cada uno de los habitantes en conseguir lo que desean y le es valioso para el desarrollo personal, familiar y comunitario.

\section{Libertades fundamentales: un análisis desde el contexto social de La Fortaleza}

Desde el panorama teórico sobre las Capacidades Humanas y sus repercusiones en la calidad de vida de una persona en el momento de poder escoger lo que desea y valora para su vida, la comunidad La Fortaleza se enfrenta a cuestionamientos que limitan esa capacidad de decidir, muestra de ello es lo expuesto por el sacerdote de la comunidad (CSO5) en el trayecto del escrito.

Ahora bien, situaciones tan individuales como lo expresado por una madre cabeza de hogar que evidencia las implicaciones de sus condiciones sociales y económicas junto con su sistema familiar: "Hay días que uno debe decidir si ir a trabajar o quedarse en casa a cuidar a mis hijos, porque tenemos que comer de algún lado y pues toca salir a buscar el pan" (CS09). Esa falta de poder decidir lo que valora y aprecia, es la baja agencia de esa madre cabeza de hogar que expresa una situación particular, pero a la vez generaliza la situación de muchas otras familias con las que convive en la comunidad. Esa agencia es dimensionada por Sen (2000) al considerar la capacidad de una persona para actuar y provocar cambios, cuyos logros pueden juzgarse en función de sus propios valores y objetivos (interpretado por Manzano y López, 2012), en este caso particular, lograr una mejor condición alimenticia para su familia.

En esa línea discursiva de análisis de la realidad social de La Fortaleza, Sen (2000) plantea las libertades fundamentales como eje principal para alcanzar el desarrollo humano en los territorios, reconociendo el papel activo de los habitantes para conseguir resultados tangibles y reales en conjunto con las diferentes organizaciones que hacen parte de una sociedad. En ese sentido, las próximas líneas protagonizarán cada una de las libertades y su relación con las expresiones narradas por los habitantes comunitarios que logran evidenciar las condiciones en las que conviven y emergen sus representaciones sociales.

En el marco de las libertades sociales, siendo ese sistema social que destina una sociedad para influir en la calidad de vida y bienestar social de las personas (Sen, 2000), el escenario comunitario contiene contradicciones sociales que afectan a los habitantes tales como los interpretados por los participantes (CS04, CS05, CS06 y CS07 en los hallazgos de la cartografía social) identificando los bajos índices educativos de los padres de familia y la poca inserción escolar de los niños, niñas y jóvenes, desempleo e informalidad, el consumo de sustancias psicoactivas y el embarazo a temprana edad, la inseguridad en general de la comunidad expresa en robos constantes, la falta de servicios públicos de la comunidad en general, y hechos referenciales como el asentamiento ilegal, consecuencias del conflicto armado y crisis colombo-venezolana. Todo lo anterior, ponen en discusión cómo las oportunidades sociales se desvanecen en la medida que los problemas sociales y estructurales hacen presencia en la comunidad.

Es así que la educación, por ejemplo, es una oportunidad social que permite expandir las libertades en general de un individuo, así lo considera autores como Ramírez, Arias y Marulanda (2016), al mencionar a la educación como un potenciador del desarrollo.

La educación es una dimensión fundamental en el crecimiento y desarrollo de un país, dada sus contribuciones al capital humano (vía productividad laboral); así como a la construcción de capacidades de los individuos. Es decir, la educación posibilita el disfrute de una vida provechosa y permite la disminución de las brechas sociales. (p. 13)

Esas capacidades de los individuos se truncan, como lo percibe el habitante (CS07), e involucra más empobrecimiento que afecta la satisfacción de las necesidades básicas y el incremento de la delincuencia en la comunidad. 
Desde esta representación, se vincula la relación del nivel educativo con el nivel de pobreza que mantiene un individuo y su sistema familiar, y que también involucra el aumento de problemáticas como la delincuencia en sus diferentes modalidades. Ello coincide con autores como Ramírez y Ramírez (2014) al referirse a los efectos de la educación en los sujetos sociales:

Sus efectos se reflejan en la calidad de vida y el bienestar de sus habitantes, logrando la reducción de los índices de pobreza, comprendida no sólo como la insuficiencia de ingresos y la no satisfacción de las necesidades básicas, sino como la carencia de las capacidades humanas. (p. 5)

Es entonces, que la oportunidad social reflejada en la educación se transforma en una oportunidad de desarrollo para el individuo que le permitirá leer, argumentar, comunicar, elegir con mayor información, ser tenida en cuenta más seriamente por otros y por los espacios construidos con su entorno (Sen, 1998).

Adicionalmente, la educación en el individuo posibilita mejoras en la convivencia social de cualquier región donde los habitantes interactúan consolidando escenarios efectivos de resolución asertiva, es en tanto que autores como Touraine y Pons (1997), Cortina (1997) e Hirmas y Eroles (2008) coinciden en privilegiar la educación como la posibilidad que fortalece el tejido social y motiva la construcción de paz en los territorios. Desde la perspectiva de Cortina (1997), el sujeto con la posibilidad educativa es: "aquel que tiene la capacidad de tomar a cualquier otra persona como un fin, y no simplemente como un medio, como un interlocutor con quien construir el mejor mundo posible" (p. 23).

En consecuencia con la línea anterior, en la comunidad La Fortaleza se expresan situaciones, en el tejido de la convivencia, como: "Pues es diferente (cómo resuelven los problemas los vecinos) porque digamos que hay una gente muy primaria que empieza así a insultar, si, o algo así, o de pronto están tomados, depende" (CS04). Es decir, en la comunidad, se evidencia una baja empatía entre los vecinos en torno a las situaciones con las que se enfrentan en su vida diaria.

Continuando el análisis, el empleo es otra oportunidad social que genera condiciones de calidad de vida estable y duradera en el tiempo para cada uno de los habitantes comunitarios, sin embargo, y como se refleja en la narrativa oral de uno de los líderes comunitarios, los habitantes en su mayoría no tienen un empleo formal y se dedican a la informalidad:

muchos viven del rebusque de vender productos en otros barrios, en una carreterilla, en una canasta en bolsos, muchos pues trabajan en lo que les salgan no entonces es frecuente ver muchas personas por ahí como des- empleadas tanto hombres como mujeres familias muy numerosas yo que la familia que menos hijos tiene son 3 de ahí para adelante 4, 5 y hasta 6 hijos. (CS05B)

Es, entonces, que se ha de considerar la influencia de la educación y el empleo en la agencia y voz de las personas para construir entornos favorables en la búsqueda de calidad de vida y aumento del desarrollo en todas sus dimensiones. Tanto es así, que la misma comunidad ha organizado sus propias oportunidades sociales cristalizadas en actores comunitarios como la biblioteca (entrevistas de la cartografía social, CS01, CS04, CS09) el comedor comunitario y la capilla católica (entrevistas de la cartografía social, CS05).

De tal forma, se reconoce a la biblioteca como una oportunidad social y comunitaria que ha posibilitado la protección de los niños, niñas y adolescentes de la comunidad, generando un apoyo a la familia en el fortalecimiento al proyecto de vida de los sujetos sociales. De ahí, viene la importancia del crecimiento comunitario al estar inclinado al empoderamiento ejercido por actores claves de la comunidad. En este sentido, el comedor comunitario se vuelve la oportunidad que tienen los grupos sociales vulnerables del contexto para acceder a unas condiciones alimenticias favorables y poder satisfacer una de las necesidades básicas del individuo, como lo indica el sacerdote de la comunidad: "se tiene un comedor para beneficios de los niños más pobres y los abuelos creo que se está completando un número de 100 personas de almuerzos diarios" (CS05).

Para finalizar el punto analítico de las oportunidades sociales y sus implicaciones en el desarrollo humano desde el contexto comunitario, se reconoce lo fundamental del sujeto comunitario para transformar su realidad social a través de su capacidad de agencia, oportunidades reconocidas en las anteriores líneas y que han permitido ser una ruta sólida para el desarrollo de la comunidad, al respecto Sen (2000) manifiesta que:

Con suficientes oportunidades sociales, los individuos pueden configurarse en realidad su propio destino $\mathrm{y}$ ayudarse mutuamente, no tienen por qué concebirse como receptores pasivos de las prestaciones de ingeniosos programas de desarrollo, existen de hecho poderosas razones para reconocer el papel positivo que desempeña la agencia libre y viable e incluso la impaciencia constructiva. (p. 28)

Desde otro ángulo de las libertades, las oportunidades políticas: "comprenden los derechos políticos que acompañan a las democracias en el sentido más amplio de la palabra" (Sen, 2000, p. 57), es decir, las posibilidades de participar en el escenario público en la consolidación del tejido democrático. Así, la realidad de esta posibilidad en la comunidad La Fortaleza se materializa con la 
organización comunitaria en la medida que los habitantes han logrado direccionar acciones para mejorar la situación social y económica a través de las Asociaciones de Mujeres y de la Junta de Acción Comunal reflejando una de las razones poderosas de Sen (2000) en una narrativa expresada:

cada asociación tiene un grupo de 30 mujeres que trabajan, tienen talleres y van funcionando muchas cosas entonces esta comunidad va creciendo y sobre todo va viendo uno el deseo de superación de muchas mujeres. (CS05)

Ligado a lo anterior, Sen (2000) ha manifestado como:

las mujeres han dejado de ser receptoras pasivas de la ayuda destinada a mejorar su bienestar y son vistas, tanto por los hombres como por ellas mismas, como agentes activos de cambio: como promotores dinámicos de transformaciones sociales que pueden alterar tanto la vida de las mujeres como la de los hombres. (p. 233)

En otras palabras, el contexto comunitario reconoce el empoderamiento de las mujeres en La Fortaleza en busca de intensificar su rol en la comunidad y en el desarrollo social de sus espacios cotidianos, no solo de las asociaciones y la junta de acción comunal, sino también del liderazgo intrínseco que ha emergido en todos los espacios de la comunidad por parte de la mujer.

En este sentido, la oportunidad política, al igual que la social y la económica, se ha ido construyendo de acuerdo con lo valorado por la comunidad y la motivación por mejorar las condiciones de su entorno, buscando en última la expansión de la calidad de vida. Sin embargo, y como carácter específico en la dinámica de la comunidad, los participantes (entrevista de la cartografía social, CS04C) manifestaron una debilidad que frena la libertad política, la cual es la búsqueda de votos, durante los procesos electorales, en la comunidad, generándose dificultades en el ejercicio transparente, crítico y responsable de las acciones democráticas.

En virtud de lo señalado, Sen (2003) considera a la democracia como valor universal, como un tesoro que se debe valorar y proteger como libertad política y, por tanto, la toma de decisiones influye el rumbo de una sociedad. Es entonces, que un riesgo que limita la libertad política de los habitantes comunitarios es el proselitismo político y la compra del voto a cambio de un interés individual implicando un bajo índice en la construcción del país que se desea.

Por otra parte, surge la libertad como seguridad protectora donde el Estado debe brindar a la ciudadanía los mecanismos necesarios para expandir sus oportunidades y posibilidades de actuación como el aumento de su agencia evitando que los ciudadanos caigan en la peor de las situaciones, por lo que "las políticas de salud, educación y empleo deben ser consideras no como mera compensación por la disfuncionalidad de una economía de mercado, sino como pilar central y vital en la reorganización social" (Lechner, 2003 p. 7). Desde esta perspectiva, en la comunidad La Fortaleza es baja la presencia de seguridad protectora al ser un asentamiento humano irregular que impide la prestación de servicios vitales para la comunidad, mientras que se conforman como barrio.

Aun así, como se identificó en la CS, la presencia de actores tanto institucionales como sociales han permitido generar procesos de fortalecimiento en el marco de la seguridad protectora como de las demás libertades instrumentales. De esta manera se describe el papel fundamental de las entidades en la comunidad a través de una expresión individual "se siente acompañado" (CS06D) que incide en la responsabilidad de la institucionalidad con la construcción de tejido social. Al respecto, autores como Durston (1999) y Canal, Gutiérrez, Trujillo y Wills (2007) encuentran una valoración positiva a esa intervención institucional (pública, privada o social) que debe buscar la formación y fortalecimiento del capital social de las comunidades permitiendo garantizar una base sostenible de cambios sociales en el tiempo, y posibilitar el empoderamiento de los habitantes comunitarios que lo conforman.

Finalmente, y no menos importante, la libertad como garantía de transparencia se proyecta en la comunidad con bajos niveles de confianza hacia algunas instituciones de orden público como la Alcaldía Municipal, al no sentir su acompañamiento y generación de condiciones hacia los habitantes comunitarios. Esas libertades de transparencia, desde una visión más comunitaria, se ve enfrentada, a su vez, con la desconfianza hacia la Junta de Acción Comunal conformada, al no sentir que albergue las necesidades de todos los habitantes sino de los más allegados compartiendo intereses en común. Si bien, es una perspectiva particular de algunos participantes, es importante fortalecer la confianza de las acciones comunitarias y públicas ligadas a la JAC.

Ahora bien, reconociendo los diálogos acaecidos, se expresaron las situaciones con matices críticos, a los que están expuestos los habitantes comunitarios de La Fortaleza y que generan un impacto directo a la libertad instrumental, y por consiguiente a la calidad de vida que construyen. En tal sentido, el enfoque Development as Freedom del Nobel Amartya Sen es una potente herramienta teórica para descubrir las condiciones a las que se enfrenta una persona, pero también las posibilidades y oportunidades que tiene para mejorar dichas situaciones. De esta forma, la Tabla 3 resume los diálogos acaecidos en este primer capítulo de análisis epistemológico y se adentra a generar un concepto social desde la visión del Trabajo Social. 
Tabla 3. Análisis sobre el desarrollo humano en la comunidad La Fortaleza

PERSPECTIVA ANÁLITICA SOBRE DESARROLLO HUMANO AMARTYA SEN

\begin{tabular}{|c|c|c|}
\hline CARTOGRAFÍA SOCIAL & CONTRADICCIONES & OPORTUNIDADES COMUNITARIAS \\
\hline \multirow{8}{*}{ LIBERTAD SOCIAL } & & Biblioteca comunitaria \\
\hline & Bajos niveles educativos & (Estrategia de lectura, deporte y música) \\
\hline & Deserción y poca cobertura educativa para los NNAJ & Comedor comunitario \\
\hline & Consumo de Sustancias Psicoactivas & (alimentación a NNAJ y personas de la Tercera Edad) \\
\hline & Embarazo a Temprana Edad & Capilla católica \\
\hline & Inseguridad & (Conciliación de los problemas de la comunidad) \\
\hline & Baja empatía vecinal & Fundación Innovación Social \\
\hline & & (Fortalecimiento comunitario) \\
\hline \multirow{3}{*}{ LIBERTAD POLÍTICA } & \multirow{2}{*}{$\begin{array}{l}\text { Individualismo de la Junta de Acción en el ejercicio } \\
\text { de intereses. }\end{array}$} & Asociación de Mujeres \\
\hline & & Junta de Acción Comunal \\
\hline & Proselitismo político y compra de votos. & \\
\hline \multirow{2}{*}{ LIBERTAD ECONÓMICA } & \multirow{2}{*}{ Desempleo e informalidad. } & Proyectos productivos locales \\
\hline & & (Pequeños negocios comerciales) \\
\hline \multirow[t]{2}{*}{$\begin{array}{l}\text { LIBERTAD COMO } \\
\text { GARANTÍAS DE } \\
\text { TRANSPARENCIA }\end{array}$} & $\begin{array}{l}\text { Falta de credibilidad en la institucionalidad. } \\
\text { (No hay presencia de actores institucionales para su } \\
\text { organización y atención prioritaria) }\end{array}$ & No se evidencia \\
\hline & $\begin{array}{l}\text { Falta de garantía sobre los Derechos Humanos } \\
\text { (Víctimas de desplazamiento, Migrantes de Venezuela). }\end{array}$ & $\begin{array}{l}\text { Universidad Francisco de Paula Santander } \\
\text { (Formación educativa) }\end{array}$ \\
\hline \multirow{4}{*}{$\begin{array}{l}\text { LIBERTAD COMO } \\
\text { SEGURIDAD } \\
\text { PROTECTORA }\end{array}$} & Falta de garantía en un mínimo vital para los habitantes & SENA \\
\hline & unitarios. & (Formación educativa- Capital semilla a propuestas \\
\hline & Carencia de servicios públicos. & de em \\
\hline & $\begin{array}{l}\text { Falta de presencia de actores institucionales claves } \\
\text { (Puestos de salud, de policía, colegios públicos, Asilos, } \\
\text { entre otros) }\end{array}$ & $\begin{array}{l}\text { Alcaldía municipal } \\
\text { (Jornadas de salud, recreación, y caracterizaciones) }\end{array}$ \\
\hline
\end{tabular}

\begin{abstract}
libertades fundamentales que impiden alcanzar y decidir lo que desean y valoran para sí y para su entorno.
CONCEPTO SOCIAL Sin embargo, se identifican posibilidades comunitarias construidas por los habitantes en aras de activar e intensificar su participación en la configuración de mejores escenarios de convivencia
\end{abstract}

Fuente: elaboración propia tomando como referencia los postulados de las libertades instrumentales de Sen (2000).

\section{Conclusiones}

La CS sirvió de catalizador no solo para identificar un ramillete de problemas sociales y estructurales, sino también cómo la comunidad La Fortaleza se organiza y busca generar formas locales para avanzar y acentuar cambios sostenibles en la vida de cada uno de sus habitantes.

El recorrido dialógico de los hallazgos, encontró en las voces de los habitantes elementos claves para reconocer su contexto social desde los actores comunitarios e institucionales presentes como sus implicaciones, así como también aquellos que son ausentes y que generan un impacto al interior del asentamiento. Ligado a ello, se vislumbraron los problemas sociales y estructurales que afectan y dinamizan la realidad de las personas, lo cual genera un panorama de las situaciones críticas a los que se enfrentan en su cotidianidad. 
En convergencia, surgen los hechos referenciales de la comunidad La Fortaleza reconociendo el territorio desde la irregularidad de su conformación, las dinámicas que conllevaron a su conformación (desplazamiento forzado, crisis socioeconómica y migración) y la presencia de condiciones de vulnerabilidad

Con lo anterior, el desarrollo no solo se mide en términos económicos o sociales, sino también en las potencialidades de base que se construyen desde la comunidad para abanderar su calidad de vida, aun así, es importante la intervención de las estructuras del Estado para fortalecer la misma calidad de vida del contexto comunitario. En este sentido, el presente escrito hizo una apuesta teórica y metodológica al configurar un análisis desde las libertades fundamentales para reconocer la realidad comunitaria de La Fortaleza y generar un concepto social desde la perspectiva de Trabajo Social, lo cual permitirá construir planes de fortalecimiento y desarrollo comunitario atendiendo a la realidad sentida y vivida de sus habitantes.

Si bien el reconocimiento del territorio no hacía parte de los objetivos directos de la investigación, sí es un componente fundamental a la hora de estudiar las representaciones sociales, pues es a partir del contexto donde se ubica una persona que le permite generar las formas de entender el mundo que le rodea. Un estudio de representación social que no parta por entender la realidad que vive un individuo en particular, no generara una comprensión holística de su estructura misma.

\section{Referencias}

Aguiló, A. J. (2009). La ciudadanía como proceso de emancipación: retos para el ejercicio de ciudadanías de alta intensidad. Astrolabio: Revista Internacional de Filosofía, 9, 13-24.

Bauman, Z. (2011). Cuarenta y cuatro cartas desde el mundo líquido. Barcelona: Paidós.

Barranco, C. (2009). Trabajo social, calidad de vida y estrategias resilientes. Portularia, 9(2), 133-145.

Canal, M., Gutiérrez, R., Trujillo, D. y Wills, E. (2007). Incidencia en el empoderamiento a comunidades atendidas por organizaciones de desarrollo y paz en Colombia. Perspectivas. Análisis de temas críticos, 25.

Cortina, A. (1997). Ciudadanos del mundo: hacia una teoría de la ciudadanía. Madrid: Alianza Editorial.

Cuñat, R. J. (2007). Aplicación de la teoría fundamentada (grounded theory) al estudio del proceso de creación de empresas. Decisiones basadas en el conocimiento $y$ en el papel social de la empresa: XX Congreso anual de AEDEM (Vol. 44). Recuperado de https://dialnet. unirioja.es/servlet/articulo?codigo $=2499458$

De Robertis, C. (2000). Respondiendo a las nuevas situaciones desde los fundamentos del Trabajo Social. Trabajo social: compromiso y equilibrio (pp. 13-34). Colegio Oficial de Diplomados en Trabajo Social de Galicia.
Durston, J. (1999). Construyendo capital social comunitario. Una experiencia de empoderamiento rural en Guatemala. Serie Políticas Sociales, N. ${ }^{\circ} 30$ (LC/L.1177), Santiago de Chile, Comisión Económica para América Latina y el Caribe (Cepal).

Giddens, A. (2007). Europa en la era global. Barcelona: Paidós Ibérica.

Hirmas, C. y Eroles, D. (2008). Convivencia democrática, inclusión y cultura de paz. Lecciones desde la práctica educativa innovadora en América Latina. Santiago de Chile: Oficina Regional de Educación de la Unesco para América Latina y el Caribe (Orealc/Unesco).

Lampis, A. (2011). ¿Qué ha pasado con la vulnerabilidad social en Colombia? Conectar libertades instrumentales y fundamentales. Sociedad y Economía, 19, 229261.

Leal, Y., Vargas, C., Villán, A., Olarte, Y. y Gutiérrez, M. (2018). Integración social de la población víctima del conflicto armado en el asentamiento humano La Fortaleza ubicado en el anillo vial occidental de la ciudad de San José de Cúcuta-Colombia. Saber, Ciencia y Libertad, 13(2), 50-64. https://doi.org/10.18041/23823240/saber.2018v13n2.4610

Lechner, N. (2003). Estado y sociedad en una perspectiva democrática. Polis. Revista Latinoamericana, 6.

Ospina, E. L. y Egea, C. (2018). Vulnerabilidad social de la población desterrada. Más allá del goce efectivo de derechos. Revista de ciencias sociales, 24(4), 38-56.

Ramírez, J., Arias, A. y Marulanda, C. (2016). Estimación del índice de agencia humana: el caso de la ciudad de San José de Cúcuta (Colombia). Revista de Economía del Caribe, 18, 92-118.

Ramírez, I. y Ramírez, J. (2014). Análisis de la deserción en el sistema educativo oficial de San José de Cúcuta (Colombia). Serie Documentos de Trabajo de Economía Regional y de Frontera. Observatorio Socioeconómico Regional de la Frontera. Universidad de Pamplona. Recuperado de http://www.unipamplona.edu.co/unipamplona/portalIG/home_72/recursos/01_general/17032015/libro_seriedoc.pdf

Restrepo, B., Velasco, A. y Preciado, B. (1999). Cartografía social. Terra Nostra, 5, 95-110.

Sampieri, R., Collado, C. y Baptista, P. (2014). Metodología de la investigación. México: McGraw-Hill.

Schütz, A. 1972. Fenomenología del mundo social. Buenos Aires: Paidós.

Sen, A. (1998). Capital humano y capacidad humana. Cuadernos de economía, 17(29), 67-72.

Sen, A. (2000). El desarrollo como libertad. Gaceta Ecológica, 55, 14-20.

Sen, A. (2003). The importance of basic education. Conferencia en Edimburgo (Escocia). 
Strauss, A. y Corbin, J. (1990). Basics of Qualitative Research: Grounded Theory, procedures and techniques. Newbury Park: Sage Publications.

Touraine, A. y Pons, H. (1997). ¿Podremos vivir juntos?: iguales y diferentes. Madrid: PPC.

Tovar, C. A. (2010). Calidad de vida: realidad y percepción. Bitácora Urbano Territorial, 17(2), 7-12.
Vélez, I., Rátiva, S. y Varela, D. (2012). Cartografía social como metodología participativa y colaborativa de investigación en el territorio afrodescendiente de la cuenca alta del río Cauca. Cuadernos de Geografia: Revista Colombiana de Geografía, 21(2), 59-73.

Urbina, J. (2016). Jóvenes universitarios en Colombia: entre la desinformación, el pesimismo y los anhelos de paz. Argumentos, 29(81). 